\title{
Multiplicity of solutions for the discrete boundary value problem involving the $p$-Laplacian
}

\author{
Abdelrachid El Amrouss \\ Mohammed I University, FSO, Oujda, Morocco, and \\ Omar Hammouti \\ Department of Mathematical Sciences, Mohammed I University, FSO, \\ Oujda, Morocco
}

\begin{abstract}
Purpose - The purpose of this paper is the study of existence and multiplicity of solutions for a nonlinear discrete boundary value problems involving the $p$-laplacian.

Design/methodology/approach - The approach is based on variational methods and critical point theory. Findings - Theorem 1.1. Theorem 1.2. Theorem 1.3. Theorem 1.4.

Originality/value - The paper is original and the authors think the results are new.

Keywords Discrete boundary value problems, $p$-Laplacian, Nontrivial solution, Variational methods

Paper type Research paper
\end{abstract}

\section{Introduction}

The main goal of the present paper is to establish the existence and multiplicity of nontrivial solutions for the following discrete nonlinear boundary value problems

$$
(P)\left\{\begin{array}{l}
-\Delta\left(\varphi_{p}(\Delta u(t-1))\right)=f(t, u(t)), t \in[1, N]_{\mathbb{Z}}, \\
u(0)=u(N+1)=0
\end{array}\right.
$$

where $N \geq 1$ is an integer, $[1, N]_{\mathbb{Z}}$ is the discrete interval $\{1, \ldots, N\}, \Delta$ is the forward difference operator defined by $\Delta u(t)=u(t+1)-u(t), \varphi_{p}(s)=|s|^{p-2} s, 1<p<\infty$ and $f:[1, N]_{\mathbb{Z}} \times \mathbb{R} \rightarrow \mathbb{R}$ is a continuous function.

By a solution of $(P)$, we mean a function $u:[0, N+1]_{\mathbb{Z}} \rightarrow \mathbb{R}$ satisfies both equations of $(P)$.

If $f(t, 0)=0$ for any $t \in[1, N]_{\mathbb{Z}}$, the constant function $u=0$ is a trivial solution of problem $(P)$. In this case, the key point is proving the existence of nontrivial solutions for $(P)$. For this purpose, we need to introduce a condition that gives us information about the behaviors of the perturbed function $f(t, x)$ or its primitive $F(t, x)$ near infinity and near zero.

We may think of $(P)$ as a discrete analogue of the following second-order $p$-Laplacian functional differential equation

\section{JEL Classification - 39A10, 34B08, 34B15, 58E30}

(C) Abdelrachid El Amrouss and Omar Hammouti. Published in Arab Journal of Mathematical Sciences. Published by Emerald Publishing Limited. This article is published under the Creative Commons Attribution (CC BY 4.0) licence. Anyone may reproduce, distribute, translate and create derivative works of this article (for both commercial and non-commercial purposes), subject to full attribution to the original publication and authors. The full terms of this licence may be seen at http:// creativecommons.org/licences/by/4.0/legalcode

The authors are grateful to the anonymous referee for carefully reading and giving valuable comments and suggestions to improve the earlier version of the paper.

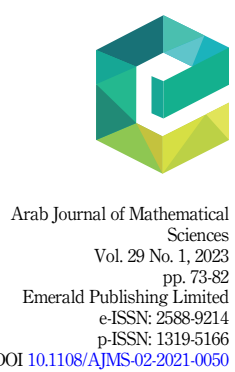




$$
\left\{\begin{array}{l}
-\frac{d}{d t}\left(\varphi_{p}\left(\frac{d u(t)}{d t}\right)\right)=f(t, u(t)), \quad t \in[0,1] \\
u(0)=u(1)=0
\end{array}\right.
$$

Let $\lambda_{1}>0$ be the first eigenvalue of the nonlinear eigenvalue problem $\left(P_{0}\right)$ corresponding to problem $(P)$

$$
\left(P_{0}\right)\left\{\begin{array}{l}
-\Delta\left(\varphi_{p}(\Delta u(t-1))\right)=\lambda \varphi_{p}(u(t)), t \in[1, N]_{\mathbb{Z}}, \\
u(0)=u(N+1)=0 .
\end{array}\right.
$$

The value of $\lambda_{1}$ is $\lambda_{1}=\min _{u \in E_{N} \backslash\{0\}} \frac{\sum_{t=1}^{N+1}|\Delta u(t-1)|^{\phi}}{\sum_{t=1}^{N}|u(t)|^{\mid}}$, where $E_{N}$ defined in (2.1) (see [1]).

The discrete analogue of the Laplacian on Riemannian manifolds, the so-called discrete Laplacian, has been studied intensively for the past few decades (see [2-7]). But, most phenomena on many cases are not expressed by the discrete Laplacian, which is known to be linear because they have a nonlinear flow governed by these intrinsic characteristics. For this reason, a nonlinear operator, called the discrete p-Laplacian, which is a generalization of the discrete Laplacian has recently been studied by many researchers in various fields, for example, dynamical systems, molecular structures, internet webs, image processing and so on (for more details, see [8-12]). Especially, many researchers have paid attention to studying boundary value problems and spectral theories for the discrete p-Laplacian (see [1, 13-19]).

As is well known, critical point theory and variational methods are powerful tools to investigate the existence of solutions of various problems.

In this paper, we shall study the existence and multiplicity of nontrivial solutions of $(P)$, via variational methods and critical point theory.

For convenience, we introduce the following notations.

$$
F_{\infty}=\lim _{|x| \rightarrow \infty} \inf \min _{t \in[1, N]_{\mathbb{Z}}} \frac{p F(t, x)}{|x|^{p}}, F^{\infty}=\lim _{|x| \rightarrow \infty} \sup _{t \in[1, N]_{\mathbb{Z}}} \frac{p F(t, x)}{|x|^{p}}, F_{0}=\lim _{x \rightarrow 0} \inf \min _{t \in[1, N]_{\mathbb{Z}}} \frac{p F(t, x)}{|x|^{p}}
$$

And we make the following conditions:

$\left(H_{1}\right)$ there exists $\eta$ with $\eta<\lambda_{1}$ such that $F^{\infty} \leq \eta$, where

$$
\lambda_{1}=\min _{u \in E_{N} \backslash\{0\}} \frac{\sum_{t=1}^{N+1}|\Delta u(t-1)|^{p}}{\sum_{t=1}^{N}|u(t)|^{p}} ;
$$

$\left(H_{2}\right)$ there exists $\delta$ with $\delta>\lambda_{N}$ such that $F_{\infty} \geq \delta$, where

$$
\lambda_{N}=\max _{u \in E_{N} \backslash\{0\}} \frac{\sum_{t=1}^{N+1}|\Delta u(t-1)|^{p}}{\sum_{t=1}^{N}|u(t)|^{p}} ;
$$

$\left(H_{3}\right) F_{0}>\lambda_{N}$;

$\left(H_{4}\right) \lim _{x \rightarrow 0} \frac{F(t, x)}{|x|^{p}}=0, \forall t \in[1, N]_{\mathbb{Z}}$;

$\left(H_{5}\right) f(t, x)$ is odd in $x$, i.e. $f(t,-x)=-f(t, x)$ for $(t, x) \in[1, N]_{\mathbb{Z}} \times \mathbb{R}$. 
The main results in this paper are the following theorems:

Theorem 1.1. $\lambda_{N}$ (defined in (1.2)) is the last eigenvalue of the nonlinear eigenvalue problem $\left(P_{0}\right)$.

Theorem 1.2. Assume that $\left(H_{1}\right)$ and $\left(H_{3}\right)$ hold, then problem $(P)$ has at least one nontrivial solution.

Theorem 1.3. Assume that $\left(H_{2}\right)$ and $\left(H_{4}\right)$ hold, then problem $(P)$ has at least two nontrivial solutions.

Theorem 1.4. Assume that $\left(H_{1}\right),\left(H_{3}\right)$ and $\left(H_{5}\right)$ hold, then problem $(P)$ has at least $2 N$ nontrivial solutions.

The rest of this paper is organized as follows. Section 2 contains some preliminary lemmas.

The main results will be proved in Sections 3 and 4.

\section{Preliminary lemmas}

In the present paper, we define a vector space $E_{N}$ by

$$
E_{N}=\left\{u:[0, N+1]_{\mathbb{Z}} \rightarrow \mathbb{R} \mid u(0)=u(N+1)=0\right\},
$$

and for any $u \in E_{N}$, define $\|u\|=\left(\sum_{t=1}^{N}|u(t)|^{p}\right)^{1 / p}$.

Equipped with \|\|$, E_{N}$ is an $N$ dimensional Banach space. In fact, $E_{N}$ is isomorphic to $\mathbb{R}^{N}$.

Let $u \in E_{N}$, we consider the functional as follows:

$$
\Phi(u)=\frac{1}{p} \sum_{t=1}^{N+1}|\Delta u(t-1)|^{p}-\sum_{t=1}^{N} F(t, u(t)) .
$$

It is easy to see that $\Phi \in C^{1}\left(E_{N}, \mathbb{R}\right)$ and its derivative $\Phi^{\prime}(u)$ at $u \in E_{N}$ is given by

$$
\Phi^{\prime}(u) \cdot v=\sum_{t=1}^{N+1}|\Delta u(t-1)|^{p-2} \Delta u(t-1) \Delta v(t-1)-\sum_{t=1}^{N} f(t, u(t)) v(t), \quad \forall v \in E_{N} .
$$

By the summation by parts formula and the fact that $v(0)=v(N+1)=0$, it follows that

$$
\begin{aligned}
& \sum_{t=1}^{N+1}|\Delta u(t-1)|^{p^{-2}} \Delta u(t-1) \Delta v(t-1)=|\Delta u(N)|^{p-2} \Delta u(N) \Delta v(N) \\
& \quad+\sum_{t=1}^{N}|\Delta u(t-1)|^{p-2} \Delta u(t-1) \Delta v(t-1)=-|\Delta u(N)|^{p-2} \Delta u(N) v(N) \\
& \quad+\left[|\Delta u(t-1)|^{p-2} \Delta u(t-1) v(t-1)\right]_{1}^{N+1}-\sum_{t=1}^{N} \Delta\left(|\Delta u(t-1)|^{p-2} \Delta u(t-1)\right) v(t) \\
& \quad=-|\Delta u(N)|^{p-2} \Delta u(N) v(N)+|\Delta u(N)|^{p-2} \Delta u(N) v(N)-|\Delta u(0)|^{p-2} \Delta u(0) v(0) \\
& \quad-\sum_{t=1}^{N} \Delta\left(|\Delta u(t-1)|^{p-2} \Delta u(t-1)\right) v(t)=-\sum_{t=1}^{N} \Delta\left(|\Delta u(t-1)|^{p-2} \Delta u(t-1)\right) v(t) .
\end{aligned}
$$


AJMS

29,1

Therefore, $\Phi^{\prime}$ can be written as

$$
\Phi^{\prime}(u) \cdot v=\sum_{t=1}^{N}\left[-\Delta\left(|\Delta u(t-1)|^{p-2} \Delta u(t-1)\right)-f(t, u(t))\right] v(t), \quad \forall v \in E_{N} .
$$

Thus, finding solutions of $(P)$ is equivalent to finding critical point of the functional $\Phi$.

Definition 2.1. Let $E$ be a real Banach space and $\Phi \in C^{1}(E, \mathbb{R})$. Recall that $\Phi$ is said to satisfy the Palais Smale $(P S)$ condition if every sequence $\left(u_{n}\right) \subset E$, such that $\Phi\left(u_{n}\right)$ is bounded and $\Phi^{\prime}\left(u_{n}\right) \rightarrow 0$ as $n \rightarrow \infty$, has a convergent subsequence. Here, the sequence $\left(u_{n}\right)$ is called a PS sequence.

Let $B_{\rho}$ denote the open ball in $E$ about 0 of radius $\rho$ and let $\partial B_{\rho}$ denote its boundary.

Lemma 2.1. (Mountain pass lemma [20]) Let $E$ be a real Banach space and $\Phi \in C^{1}(E, \mathbb{R})$ satisfy the (PS) condition. If $\Phi(0)=0$ and

$\left.\sigma_{1}\right)$ there exist constants $\rho, \alpha>0$ such that $\left.\Phi\right|_{\partial B_{\rho}} \geq \alpha$,

$\left.\sigma_{2}\right)$ there exist $e \in E B_{\rho}$ such that $\Phi(e) \leq 0$.

Then $\Phi$ possesses a critical value $c \geq$ a given by $c=\inf _{g \in \Gamma \in[0,1]} \Phi(g(s))$,
where

$$
\Gamma=\{g \in C([0,1], E) / g(0)=0, g(1)=e\} .
$$

Lemma 2.2. (see [21]) Let Ebe a realBanach space and $\Phi \in C^{1}(E, \mathbb{R})$ be even, bounded from below, and satisfy the (PS) condition. Suppose that $\Phi(0)=0$ and there is a set $\Omega \subset E$ such that $\Omega$ is homeomorphic to $S^{n-1}$ by an odd map and $\sup \Phi(u)<0$, where $S^{n-1}$ is the $n-1$ dimensional unit sphere. Then, $\Phi$ has at least $n$ disjoint pairs of nontrivial critical points.

\section{Eigenvalue problem}

We consider the nonlinear eigenvalue problem $\left(P_{0}\right)$ corresponding to problem $(P)$ :

$$
\left(P_{0}\right)\left\{\begin{array}{l}
-\Delta\left(\varphi_{p}(\Delta u(t-1))\right)=\lambda \varphi_{p}(u(t)), t \in[1, N]_{\mathbb{Z}}, \\
u(0)=u(N+1)=0,
\end{array}\right.
$$

Definition 3.1. $\lambda \in \mathbb{R}$ is called eigenvalue of $\left(P_{0}\right)$ if there exists $u \in E_{N} \backslash\{0\}$ such that:

$$
\sum_{t=1}^{N+1} \varphi_{p}(\Delta u(t-1)) \Delta v(t-1)=\lambda \sum_{t=1}^{N} \varphi_{p}(u(t)) v(t), \quad \forall v \in E_{N} .
$$

Proposition 3.1. (see [22]) Let $E$ be a real Banach space, $G, J \in C^{1}(E, \mathbb{R})$ and a set of constraints

$S=\{u \in E \mid G(u)=0\}$. Suppose that for any $u \in S, G^{\prime}(u) \neq 0$ and there exists $u_{0} \in S$ such that $J\left(u_{0}\right)=\inf _{u \in S} J(u)$. Then there is $\lambda \in \mathbb{R}$ such that $J^{\prime}\left(u_{0}\right)=\lambda G^{\prime}\left(u_{0}\right)$.

Proof of Theorem 1.1. Put $J(u)=\sum_{t=1}^{N+1}|\Delta u(t-1)|^{p}, G(u)=\sum_{t=1}^{N}|u(t)|^{p}-1$, and

$$
S=\left\{u \in E_{N} \mid G(u)=0\right\}=\left\{u \in E_{N} \mid\|u\|=1\right\} .
$$


It is easy to see that $G^{\prime}(u) \neq 0$ for any $u \in S$.

If the set $S$ is compact and $J$ is continuous on $S$, then there exists $u_{N} \in S$ such that

$$
J\left(u_{N}\right)=\max _{u \in S} J(u)=\lambda^{\prime} .
$$

Discrete boundary value problem

Thus,

$$
-J\left(u_{N}\right)=\min _{u \in S}(-J(u))=-\lambda^{\prime}
$$

Clearly $\lambda^{\prime}>0$. From the Proposition 3.1, there exists $\lambda_{N}$ such that

$$
J^{\prime}\left(u_{N}\right)=\lambda_{N} G^{\prime}\left(u_{N}\right) \text {. }
$$

Which means that

$$
-\Delta\left(\varphi_{p}\left(\Delta u_{N}(t-1)\right)\right)=\lambda_{N} \varphi_{p}\left(u_{N}(t)\right), \quad t \in[1, N]_{\mathbb{Z}} .
$$

Multiplying (3.1) by $u_{N}$ in the sense of inner product, we obtain

$$
\sum_{t=1}^{N+1}\left|\Delta u_{N}(t-1)\right|^{p}=\lambda_{N} \sum_{t=1}^{N}\left|u_{N}(t)\right|^{p}
$$

i.e.

$$
J\left(u_{N}\right)=\lambda_{N}\left\|u_{N}\right\|^{p}=\lambda_{N} .
$$

Therefore, $\lambda^{\prime}=\lambda_{N}$ is an eigenvalue of the problem $\left(P_{0}\right)$.

Thus, we have

$$
\begin{aligned}
\lambda_{N} & =\max _{u \in S} \sum_{t=1}^{N+1}|\Delta u(t-1)|^{p} \\
& =\max _{u \in E_{N} \backslash\{0\}} \sum_{t=1}^{N+1}\left|\Delta \frac{u(t-1)}{\|u\|}\right|^{p} \\
& =\max _{u \in E_{N} \backslash\{0\}} \frac{\sum_{t=1}^{N+1}|\Delta u(t-1)|^{p}}{\sum_{t=1}^{N}|u(t)|^{p}} .
\end{aligned}
$$

If $\lambda$ is an eigenvalue of the problem $\left(P_{0}\right)$, then there exists $u \in E_{N} \backslash\{0\}$ such that:

$$
\sum_{t=1}^{N+1} \varphi_{p}(\Delta u(t-1)) \Delta v(t-1)=\lambda \sum_{t=1}^{N} \varphi_{p}(u(t)) v(t), \quad \forall v \in E_{N} .
$$

In particular for $v=u$, we get $\lambda=\frac{\sum_{t=1}^{N+1}|\Delta u(t-1)|^{p}}{\sum_{t=1}^{N}|u(t)|^{\phi}}$.

So, we deduce that $\lambda_{1} \leq \lambda \leq \lambda_{N}$.

Then, $\lambda_{N}$ is the last eigenvalue of the problem $\left(P_{0}\right)$.

The proof of Theorem 1.1 is completed. 


\section{Proofs of the main results}

Proof of Theorem 1.2. Since $F^{\infty} \leq \eta$, there exists $R_{1}>0$ such that

$$
\left.\frac{p F(t, x)}{|x|^{p}} \leq \eta+\varepsilon \quad \text { for } \quad(t,|x|) \in[1, N]_{\mathbb{Z}} \times\right] R_{1},+\infty[,
$$

where $0<\varepsilon<\lambda_{1}-\eta$, i.e.

$$
\left.F(t, x) \leq \frac{1}{p}(\eta+\varepsilon)|x|^{p} \quad \text { for }(t,|x|) \in[1, N]_{\mathbb{Z}} \times\right] R_{1},+\infty[.
$$

Then, by (4.1) and the continuity of $x \rightarrow F(t, x)$, there exists $c_{1}>0$ such that

$$
F(t, x) \leq \frac{1}{p}(\eta+\varepsilon)|x|^{p}+c_{1}, \quad \forall(t, x) \in[1, N]_{\mathbb{Z}} \times \mathbb{R} .
$$

According to (1.1), we have

$$
\sum_{t=1}^{N+1}|\Delta u(t-1)|^{p} \geq \lambda_{1}\|u\|^{p} .
$$

Using (4.2) and (4.3), we obtain

$$
\begin{aligned}
\Phi(u) & \geq \frac{1}{p} \lambda_{1}\|u\|^{p}-\frac{1}{p}(\eta+\varepsilon) \sum_{t=1}^{N}|u(t)|^{p}-c_{1} N \\
& \geq \frac{1}{p}\left[\lambda_{1}-(\eta+\varepsilon)\right]\|u\|^{p}-c_{1} N .
\end{aligned}
$$

Since $\varepsilon<\lambda_{1}-\eta$, then $\Phi(u) \rightarrow \infty$ as $\|u\| \rightarrow \infty$. Thus, $\Phi$ is coercive and bounded from below, hence there is a minimum point of $\Phi$ at some $u_{0} \in E_{N}$ i.e. $\Phi\left(u_{0}\right)=\inf _{u \in E_{N}} \Phi(u)$, which is a critical point of $\Phi$ and in turn is a solution of problem $(P)$.

From $\left(H_{3}\right)$, there exists $\rho_{1}>0$ such that

$$
\frac{p F(t, x)}{|x|^{p}} \geq F_{0}-\varepsilon, \quad \text { for }(t,|x|) \in[1, N]_{\mathbb{Z}} \times\left[0, \rho_{1}\right],
$$

where $0<\varepsilon<F_{0}-\lambda_{N}$, i.e.

$$
F(t, x) \geq \frac{1}{p}\left(F_{0}-\varepsilon\right)|x|^{p} \quad \text { for }(t,|x|) \in[1, N]_{\mathbb{Z}} \times\left[0, \rho_{1}\right] .
$$

Put

$$
\Omega=\left\{u \in E_{N} \mid\|u\|=\rho_{1}\right\}
$$

For any $u \in \Omega$,

$$
|u(t)| \leq\|u\|=\rho_{1}, \quad \forall t \in[1, N]_{\mathbb{Z}} .
$$


From (1.2), we get

$$
\sum_{t=1}^{N+1}|\Delta u(t-1)|^{p} \leq \lambda_{N}\|u\|^{p} .
$$

Discrete boundary value problem

Combining the preceding inequality and (4.4), we have

$$
\begin{aligned}
\Phi(u) & \leq \frac{1}{p} \lambda_{N}\|u\|^{p}-\frac{1}{p}\left(F_{0}-\varepsilon\right)\|u\|^{p} \\
& =\frac{1}{p}\left[\lambda_{N}-\left(F_{0}-\varepsilon\right)\right] \rho_{1}^{p}<0 .
\end{aligned}
$$

Thus, we obtain

$$
\sup _{u \in \Omega} \Phi(u)<0 .
$$

Hence,

$$
\Phi\left(u_{0}\right)=\inf _{u \in E_{N}} \Phi(u) \leq \inf _{u \in \Omega} \Phi(u) \leq \sup _{u \in \Omega} \Phi(u)<0 .
$$

So problem $(P)$ has at least one nontrivial solution.

The proof of Theorem 1.2 is completed.

Proof of Theorem 1.3. From the condition $\left(H_{4}\right)$, for $\varepsilon=\frac{\lambda_{1}}{2 p}$ there exists $\rho_{2}>0$ such that:

$$
|F(t, x)| \leq \frac{\lambda_{1}}{2 p}|x|^{p} \quad \text { for }(t,|x|) \in[1, N]_{\mathbb{Z}} \times\left[0, \rho_{2}\right] .
$$

Let $u \in E_{N}$ with $\|u\| \leq \rho_{2}$, then $|u(t)| \leq \rho_{2}, \quad \forall t \in[1, N]_{\mathbb{Z}}$.

Thus, we have

$$
\begin{aligned}
\Phi(u) & \geq \frac{\lambda_{1}}{p}\|u\|^{p}-\frac{\lambda_{1}}{2 p}\|u\|^{p} \\
& \geq \frac{\lambda_{1}}{2 p}\|u\|^{p} .
\end{aligned}
$$

Take $\alpha=\frac{\lambda_{1}}{2 p} \rho_{2}^{p}>0$. Therefore,

$$
\Phi(u) \geq \alpha>0, \quad \forall u \in \partial B_{\rho_{2}} .
$$

At the same time, we have also proved that there exist constants $\alpha>0$ and $\rho_{2}>0$ such that $\left.\Phi\right|_{\partial B_{\rho_{2}}} \geq \alpha$. That is to say, $\Phi$ satisfies the condition $\sigma_{1}$ of the mountain pass lemma.

For our setting, clear $\Phi(0)=0$. In order to exploit the mountain pass lemma in critical point theory, we need to verify all other conditions of the mountain pass lemma.

According to the condition $\left(H_{2}\right)$, there exists $R_{2}>0$ such that

$$
\left.\frac{p F(t, x)}{|x|^{p}} \geq \delta-\varepsilon \quad \text { for } \quad(t,|x|) \in[1, N]_{\mathbb{Z}} \times\right] R_{2},+\infty[,
$$


AJMS

29,1

where $0<\varepsilon<\delta-\lambda_{N}$, i.e.

$$
\left.F(t, x) \geq \frac{1}{p}(\delta-\varepsilon)|x|^{p} \quad \text { for }(t,|x|) \in[1, N]_{\mathbb{Z}} \times\right] R_{1},+\infty[.
$$

Then, by (4.10) and the continuity of $x \rightarrow F(t, x)$, there exists $c_{2}>0$ such that

$$
F(t, x) \geq \frac{1}{p}(\delta-\varepsilon)|x|^{p}-c_{2}, \quad \forall(t, x) \in[1, N]_{\mathbb{Z}} \times \mathbb{R} .
$$

Now, using again (4.6) and (4.11), it follows that,

$$
\begin{aligned}
\Phi(u) & \leq \frac{\lambda_{N}}{p}\|u\|^{p}-\frac{1}{p}(\delta-\varepsilon)\|u\|^{p}+c_{2} N \\
& \leq \frac{1}{p}\left[\lambda_{N}-(\delta-\varepsilon)\right]\|u\|^{p}+c_{2} N .
\end{aligned}
$$

Consequently, since $\varepsilon<\delta-\lambda_{N}$, we have

$$
\Phi(u) \rightarrow-\infty, \text { as }\|u\| \rightarrow \infty .
$$

Thus, we can choose $\bar{u}$ large enough to ensure that $\Phi(\bar{u})<0$, that is, there exists $e=\bar{u} \in E \backslash B_{\rho_{2}}$ such that $\Phi(e)<0$.

From (4.12), $\Phi$ is anti-coercive, hence for any PS sequence $\left(u_{n}\right)$ is bounded. In view of the fact that the dimension of $E_{N}$ is finite, we see that $\Phi$ satisfies the $(P S)$ condition.

By the mountain pass lemma, $\Phi$ possesses a critical value $c \geq \alpha=\frac{\lambda_{1}}{2 p} \rho_{2}^{p}>0$,

where $c=\inf _{g \in \Gamma} \max _{s \in[0,1]} \Phi(g(s))$ and $\Gamma=\{g \in C([0,1], E) \mid g(0)=0, g(1)=\bar{u}\}$.

Let $u_{1} \in E_{N}$ be a critical point associated to the critical value $c$ of $\Phi$, i.e. $\Phi\left(u_{1}\right)=c$.

Hence, $u_{1}$ is a nontrivial solution of problem $(P)$.

Since $\Phi$ is anti-coercive and bounded from above, there is a maximum point of $\Phi$ at some $u_{2} \in E_{N}$,

i.e. $\Phi\left(u_{2}\right)=\sup _{u \in E_{N}} \Phi(u)$.

Using the preceding equality and (4.9), we obtain

$$
\Phi\left(u_{2}\right)=\sup _{u \in E_{N}} \Phi(u) \geq \sup _{u \in \partial B_{\rho_{2}}} \Phi(u)>0 .
$$

Hence $u_{2}$ is a nontrivial solution of problem $(P)$.

If $u_{1} \neq u_{2}$, then we have two nontrivial solutions: $u_{1}$ and $u_{2}$.

Otherwise, suppose $u_{1}=u_{2}$, then $\inf _{g \in \Gamma_{s} \in[0,1]} \Phi(g(s))=\sup _{u \in E_{N}} \Phi(u)$.

Therefore, we have $\Phi\left(u_{1}\right) \leq \max _{s \in[0,1]} \Phi(g(s)) \leq \Phi\left(u_{2}\right), \quad \forall g \in \Gamma$.

Since $u_{1}=u_{2}$, we deduce that $\Phi\left(u_{1}\right)=\max _{s \in[0,1]} \Phi(g(s)), \forall g \in \Gamma$.

The continuity of $\Phi(g(s))$ with respect to $s, \Phi(0)=0$ and $\Phi(\bar{u})<0$ implies that there exists $\left.s_{1} \in\right] 0,1\left[\right.$ such that $\Phi\left(u_{1}\right)=\Phi\left(g\left(s_{1}\right)\right)$. Choose $g_{2}, g_{3} \in \Gamma$ such that

$$
\left\{g_{2}(s) \mid s \in\right] 0,1[\} \cap\left\{g_{3}(s) \mid s \in[0,1]\right\}=\varnothing,
$$

then there exists $\left.s_{2}, s_{3} \in\right] 0,1[$ such that

$$
\Phi\left(g_{2}\left(s_{2}\right)\right)=\Phi\left(g_{3}\left(s_{3}\right)\right)=\Phi\left(u_{1}\right)=\max _{s \in[0,1]} \Phi(g(s)) .
$$

Thus, we get two different critical points of $\Phi$ on $E_{N}$ denoted by $v_{2}=g_{2}\left(s_{2}\right), v_{3}=g_{3}\left(s_{3}\right)$ that are nontrivial solutions of problem $(P)$. 
The proof is completed.

Proof of Theorem 1.4. Let $\Phi$ be defined by (2.2). Then, it is clear that $\Phi(0)=0$ and $\Phi$ is even by $\left(H_{5}\right)$.

From the proof of Theorem 1.2, $\Phi$ is bounded from below, coercive and any PS sequence $\left(u_{n}\right)$ is bounded. In view of the fact that the dimension of $E_{N}$ is finite, we see that $\Phi$ satisfies the $(P S)$ condition.

Let $S^{N-1}$ be the unit sphere in $\mathbb{R}^{N}$ and define $T: \Omega \rightarrow S^{N-1}$ by

$$
T(u)=\frac{1}{\rho_{1}} u,
$$

where $\Omega$ (defined in (4.5)).

Then, $T$ is an odd homeomorphism between $\Omega$ and $S^{N-1}$, and $\sup _{u \in \Omega} \Phi(u)<0$ (see (4.7)).

Hence, all the conditions of Lemma 2.2 are satisfied, so $\Phi$ has at least $2 N$ nontrivial critical points, which are nontrivial solutions of problem $(P)$.

This completes the proof of Theorem 1.4.

\section{References}

1. Agarwal RP, Perera K, O’Regan D, Multiple positive solutions of singular discrete $p$-Laplacian problems via variational methods, Adv Differ Equ. 2005; 2(2): 93-9.

2. Bendito E, Carmona A, Encinas AM, Solving Dirichlet and Poisson problems on graphs by means of equilibrium measures, Eur J Combin. 2003; 24(4): 365-75.

3. Bondy JA, Hemminger RL, Graph reconstruction—a survey, J Graph Theory. 1977; 1(3): 227-68.

4. Curtis EB, Ingerman D, Morrow JA, Circular planar graphs and resistor networks, Linear Algebra Appl. 1998; 283(1-3): 115-50.

5. Curtis EB, Morrow JA, The Dirichlet to Neumann map for a resistor network, SIAM J Appl Math. 1991; 51(4): 1011-29.

6. Curtis E, Mooers E, Morrow JA, Finding the conductors in circular networks from boundary measurements, RAIRO Model. Math Anal Numer. 1994; 28(7): 781-814.

7. Elmoataz A, Lezoray O, Bougleux S, Nonlocal discrete regularization on weighted graphs: a framework for image and manifold processing, IEEE Trans Image Process. 2008; 17(7): 1047-60.

8. Agarwal RP, O'Regan D, Stank S. An existence principle for nonlocal difference boundary value problems with $\phi$-Laplacian and its application to singular problems. Adv Differ Equ. 2008; 14: 154302.

9. Ha SY, Levy D, Kinetic and fluid models for phototaxis, Discrete Contin Dyn Syst Ser B. 2009; 12(1): 77-108.

10. Ha SY, Lee K, Levy D, Emergence of time-asymptotic flocking in a stochastic Cucker-Smale system, Commun Math Sci. 2009; 7(2): 453-69.

11. Trinajstic N, Chemical graph theory, $2^{\text {nd }}$ ed., Boca Raton, FL; CRC Press: 1992.

12. Ta V, Bougleux S, Elmoataz A, Lezoray O, Nonlocal anisotropic discrete regularization for image, data filtering and clustering, Tech. Rep. Caen; University of Caen: 2007.

13. Amghibech S, Bounds for the largest p-Laplacian eigenvalue for graphs, Discrete Math. 2006; 306(21): 2762-71.

14. Amghibech S, On the discrete version of Picone's identity, Discrete Appl Math. 2008; 156(1): 1-10.

15. Candito P, Giovannelli N, Multiple solutions for a discrete boundary value problem involving the p-Laplacian, Comput Math. Appl. 2008; 56: 959-64. 
AJMS

29,1

82

16. Holopainen I, Soardi PM, $p$-harmonic functions on graphs and manifolds, Manuscripta Math. 1997; 94(1): 95-110.

17. Holopainen I, Soardi PM, A strong Liouville theorem for $p$-harmonic functions on graphs, Ann Acad Sci Fenn Math. 1997; 22(1): 205-26.

18. Jiang L, Zhou Z, Three solutions to Dirichlet boundary value problems for $p$-Laplacian difference equations, Adv Differ Equ. 2008; 10: 345916.

19. Kim SW, Lee YH, Positive p-harmonic functions on graphs, Bull Korean Math. Soc. 2005; 42 (2): 421-32.

20. Rabinowitz PH, Minimax methods in critical point theory with applications to differential equations. Providence, RI, New York, NY; American Mathematical Society: 1986.

21. Graef JR, Kong L, Wang M, Multiple solutions to a periodic boundary value problem for a nonlinear discrete fourth order equation. Adv Dynamical Syst Appl. 2013; 8(2): 203-15. 0973-5321.

22. Kavian O, Introduction à la théorie des points critiques. Paris; Springer-Verlag France: 1993.

\section{Further reading}

23. Agarwal RP, Difference equations and inequalities, theory, methods, and applications, $2^{\text {nd }}$ ed. New York, NY: Marcel Dekker; 2000.

\section{Corresponding author}

Omar Hammouti can be contacted at: omar.hammouti.83@gmail.com

For instructions on how to order reprints of this article, please visit our website:

www.emeraldgrouppublishing.com/licensing/reprints.htm

Or contact us for further details: permissions@emeraldinsight.com 